\title{
Achieving Excellence in Indian Engineering Education through ContinuousQuality Improvement
}

\author{
Pulok Ranjan Mohanta \\ Department of Mechanical Engineering , Laxmi Institute of Technology, Sarigam, Gujarat \\ pulokmohanta@gmail.com
}

\begin{abstract}
The engineering education is a confluence of research, experiment and experience. Engineeringeducation is essential to improve the technical manpower of a country. Technical manpower isthe key to the industrial growth and development of a country. In the recent years though thenumber of the engineering graduates being introduced to the job market increased rapidly everyyear, the statistics of unemployable workforce also have the similar trend. The quality of thegraduates and their employability is determined solely by the education quality they received.This unemployment scenario resulted in shifting of interest of the potential school students fromengineering courses to other courses which are relatively cheaper. As a result of which a largenumber of seats are lying vacant in a number of engineering institutions. Applications for closureof the college/ courses or reducing sanctioned intake are increasing at AICTE. In this scenario

where survivals of the colleges are at stack, the quality of education an engineering instituteoffers will only be the sole rescue. In the current paper a conceptual model for improving qualityof engineering education through continuous quality improvement is highlighted.
\end{abstract}

Keywords: Engineering education, quality, CQI, outcome based education.

\footnotetext{
Pulok Ranjan Mohanta

Department of Mechanical Engineering, Laxmi Institute of Technology, Sarigam, Gujarat.

pulokmohanta@gmail.com
}

\author{
1. Current scenario of the Engineering Education in \\ India.
}

Technical education at all levels in India is witnessing a consistent growth pattern marked by the setting up of new Institutions and the improvement of the existing ones in tune with the qualityassurance norms set by the accreditation agencies (AICTE). The beginning of formal TechnicalEducation in India can be dated back to the mid 19 th century. The major policy initiatives in the pre-

independence period included appointment of the Indian Universities Commission in 1902, issue of the Indian Education policy resolution in 1904 and the Governor General's policystatement of 1913 stressing the importance of Technical Education, the establishment of IISc. InBangalore, Institution for Sugar, Textile and Leather Technology in Kanpur, N.C.E. in Bengal in1905 and Industrial schools in several provinces. The growth of Technical Education beforeindependence in the Country has been very slow. The number of Engineering Colleges andPolytechnics (including Pharmacy and Architecture Institutions) in 1947 was 44 and 43respectively with an intake capacity of 3200 and 3400 respectively. In recent years Indiawitnessed a tremendous growth in number of intake in engineering from 653290 in 2007-08 to1903722 in 2014-15 (AICTE). Polytechnic students' intake also shows the similar figure with417923 in 2007-08 to 1308008 in 2014-15.Unfortunately, though the quantum of engineering graduates produced is high, the failure toadopt and adhere to international quality standards by the engineering institutions has resulted inproducing substandard graduates who terribly lack in the required skill sets as demanded byindustry. This, while resulting in critical shortage of employable and skilled engineeringstudents, also created a vast pool of unemployable workforce. 


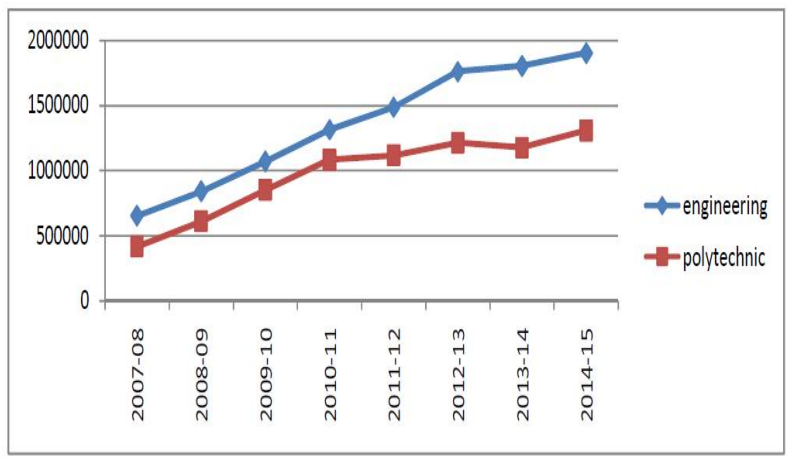

Fig.1 Growth of intake in engineering and polytechnic intake (Source AICTE)

\section{Continuous Quality Improvement}

Continuous Quality Improvement (CQI) is well described in its simplest form as a quality management process that encourages all team members to continuously ask the questions, "How are we doing?" and "Can we do it better?"(Edwards et al 2008). More specifically, can we do it more efficiently? Can we be more effective? Can we have a greater impact? Moreover it is a culture that that seeks improvements in its practice, operation and achievements. Besides creating this inquisitive CQI culture in an institution, the key to any CQI initiative is using a structured planning approach to evaluate the current practice processes and improve systems and processes to achieve the desired outcome and vision for the desired future state. Tools commonly used in CQI include strategies that enable team members to assess and improve their performance in providing the desired services. The CQI plan identifies the desired organizational outcome and the evaluation strategies that enable the team to determine if they are achieving that outcome. The team also intervenes, when needed, to adjust the CQI plan based on continuous monitoring of progress through an adaptive, real-time feedback loop.

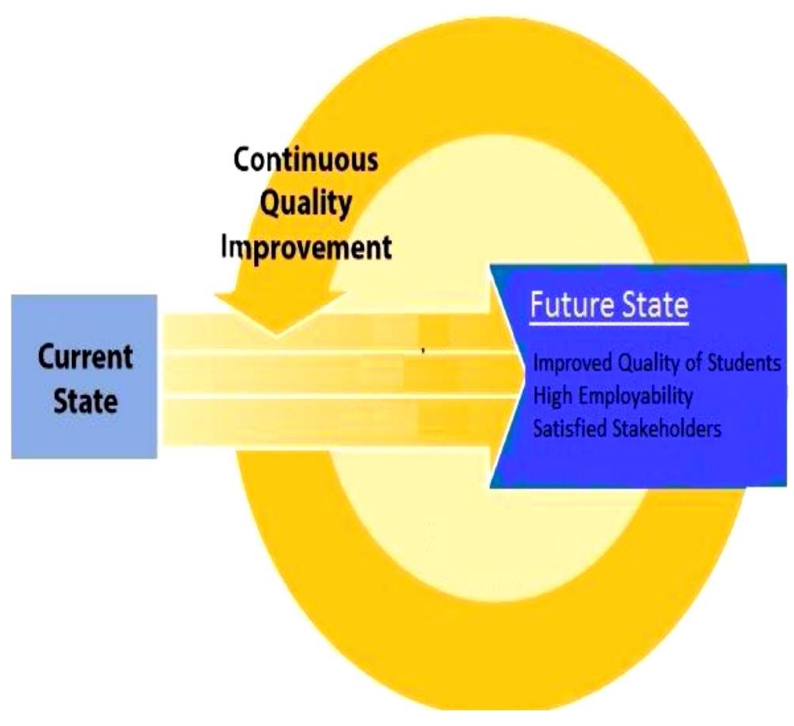

Fig. 2 CQI cycle

\section{Components of CQI}

In undertaking any CQI initiative, a practice must consider these components:

\section{A. Structure}

Structure includes the technological, human, physical, and financial assets a practice possesses to carry out its work. CQI examines the characteristics (e.g., number, mix, location, quality, and adequacy) of resources, staff and consultant, physical space, and financial resources.

\section{B. Process}

The activities, workflows, or task(s) carried out to achieve an output or outcome are considered process. Although CQI strategies in the literature focus more commonly on teaching learning, CQI also applies to administrative processes.

\section{Outcome}

Outcomes are the end result of the service and a change in the quality of the students. Desired changes in the employability can also be considered outcomes.

\section{Feedback Loop}

In Fig. 3 a feedback loop between the output/outcome and the CQI initiative represents its cyclical, iterative nature. Once a change to the structure and process is implemented, a practice must determine whether it achieved the intended outcome and, if not, what other changes could be considered. If the outcome is achieved, the practice could determine how to produce an even better outcome or achieve it more efficiently and with less cost.

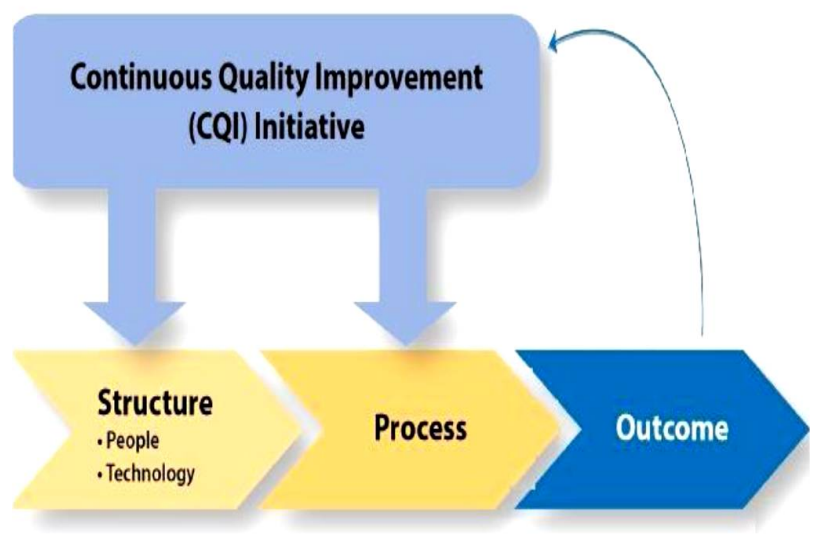

Fig. 3 Feedback loop for CQI

\section{Improvement of Components of CQI}

There should be an improvement of the components of CQI is required and that can be done by various process.

\section{A. Improving Structure}

The most important component in any education system is the teacher. The performance of teacher determines the success level of all the education or training processes. So it 
becomes necessary for any engineering institute to induct adequately qualified and motivated teachers. Proper HR policies must also be implemented to retain the faculty and keep their motivation for teaching up. Enough opportunity must also be provided to the teachers for their skill and knowledge enhancement. They must also be encouraged to undergo need based trainings as and when organized by premier institutes of the country like IITs, NITs etc. In the present era technology also plays a vital role in knowledge sharing and acquiring process. The engineering institutes must also consider up gradation to the latest technology periodically. This will enable the students and teachers to get exposure to latest trends and broaden their knowledge base. It will also help in enhancing the effectiveness of teaching learning process. One can access the teachings from experts from different universities from different locations easily. Various courses are also offered by premiere institutes under MOOCs, NPTEL which every institute can access. The teachers and students must be encouraged to take the advantage. For Indian higher education system, MOOCs actually would provide a very convenient model to impart quality education across a large number of private institutions (Rao et al 2015). As far as possible the engineering colleges must develop proper laboratory infrastructure to enable students and teachers have the proper understanding of the concepts that need experimental observations. The institutes must collaborate with the industries for industrial visits and expert lectures by the practicing engineers to make the students aware of the ongoing trends. The continuous improvement of structure can be summarized by the Fig. 4 inspired from International Organization for Standardization, 2011.The ISO 9000 family - core standards.

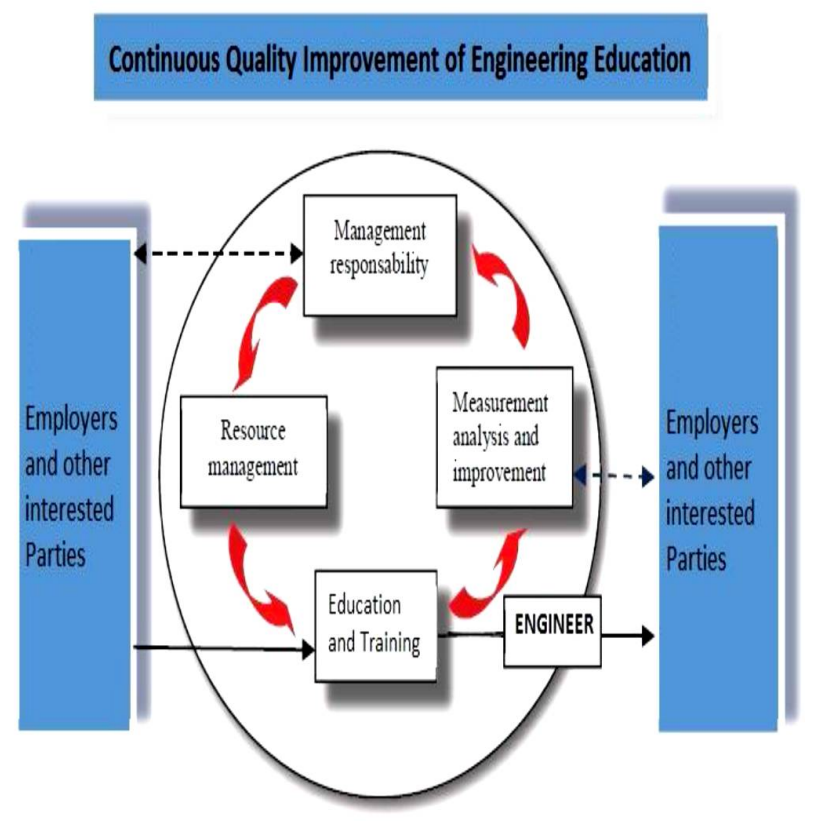

Fig. 4 The ISO 9000 process approach
Within engineering education institutions Continuous quality improvement in its educationprocess is achieved by measuring the quality of processes and outcomes. It is necessary tointroduce and use quantitative and qualitative indicators, and by comparing it to stakeholders satisfaction. To continuously improve both research and educational products and services one should increase the efficiency and effectiveness of the quality of the university processes, and permanently evaluate the process outcomes and criteria used to analyze the results. Another possible way of evaluation is by conducting audits and/or benchmarking processes where one can obtain data to analyze the process and give decision. This type of factual approach is possible when data is available and reliable. An engineering education institution should developa system specially designed for collecting and assessing the necessary data. To make appropriate and sound decisions the data and the information should be clear and accessible and should be first analyzed The factual approach in decision making is vital as all decisions are based on data rather than on simple assumptions [4].It is very important that any higher education institution identifies its customers and stakeholders (students, professors, parents, employees, etc.) in order to understand their needs and expectations [5]. Following the steps of continuous improvement as described in Fig. 4 the higher education institution aims at best fulfilling their needs and satisfying their requirements. The similarity with Six Sigma relies on the consideration of this principle, customer orientation, as a prerequisite of the success of an organization. Involvement of people brings active participation at various activities forward, positive attitudes regarding their creative role and responsible attitudes towards solution finding. The involvement of people in different activities and processes in a higher education institution should be increased as knowledge creation, dissemination and utilization are very important. The quality education system is process based according to the revised version of ISO 9000. Process components that are to be taken into account are the inputs and outputs of the teaching learning and research processes. A clear evaluation of the risks and effects of the performed processes upon the various stakeholders is to be made. Engineering education is viewed as a network of processes, where the existence of resources and their efficient use play an important role in achieving quality outcomes and quality educational services. Proper training and development of

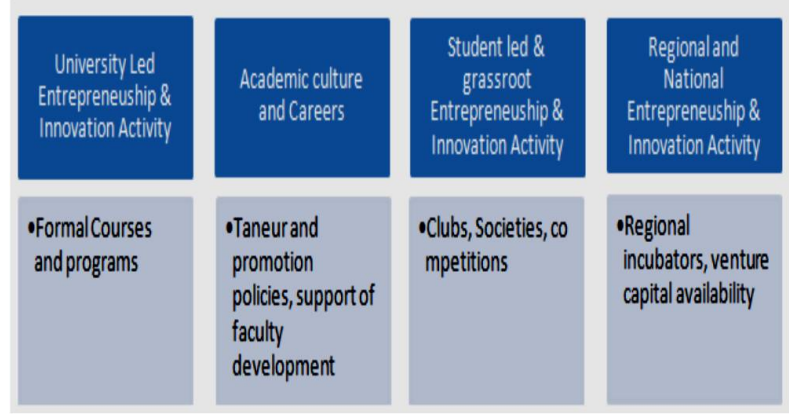

Fig. 5 Integration of innovation and entrepreneurship

\section{B. Process Improving}


the existing faculty and addition of expert from different background if necessary will definitely ensure the improvement in education process of the engineers. The institutions must be in continuous communication with $R \& D$ organizations and industries for the up gradation of their knowledge and to find out the knowledge elements that are necessary to be imparted to the students. Engineering institution must try to convert their programs into residential ones. This will enable the students to reduce travelling time and collaborate with the teachers as and when required. The environment so created will foster a high quality engineer production system. The other important elements that can be considered in improving process are integrating innovation and entrepreneurship. The strategies for this integration are briefly illustrated in the Fig. 5.

\section{Outcome Improvement}

The efforts and investment the engineering institutions put in improving structure and process is solely to obtain the desired outcomes. The following efforts must put by the institutions to improve the outcome of the engineering courses.

1) Integration of AICTE reform efforts driven by a commitment to high academic standards.

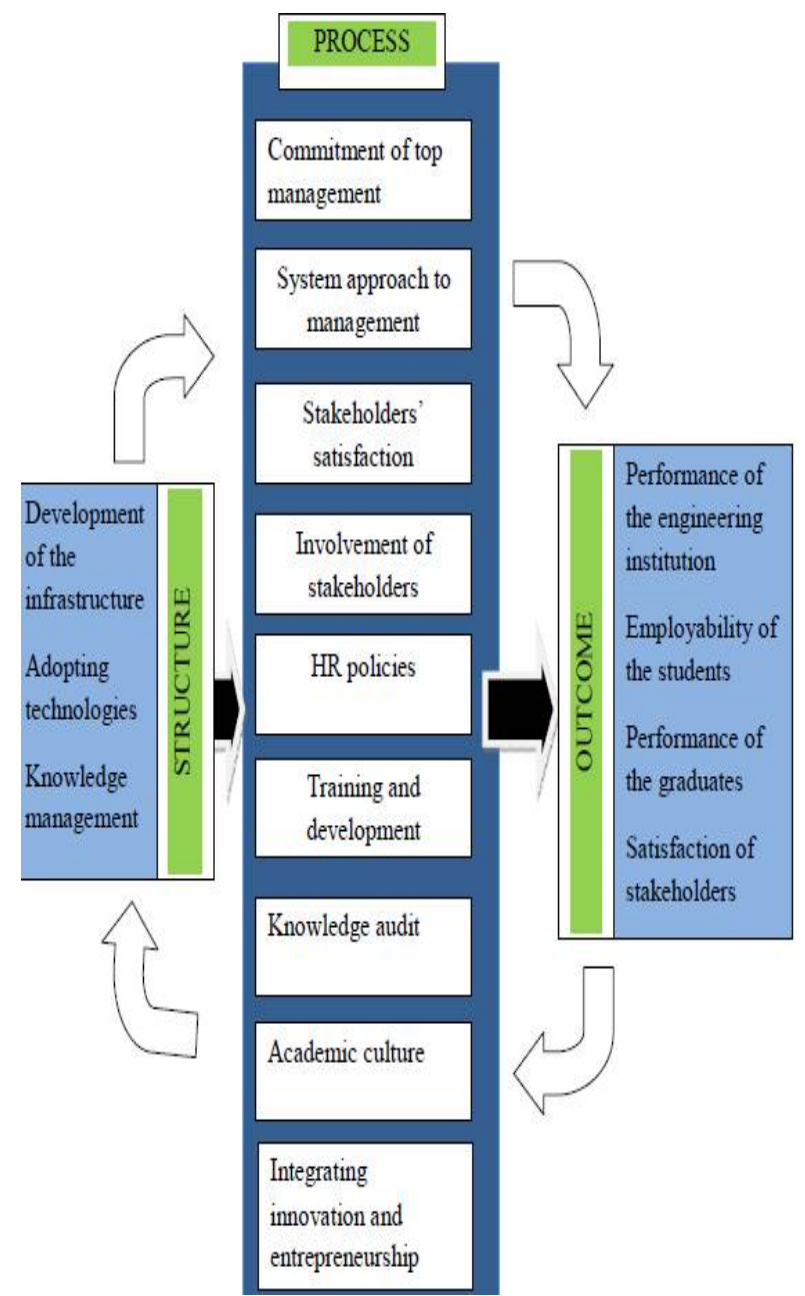

Fig. 6 Continuous Quality Improvement Cycle in a nutshell

2) Adoption of internationally recognized industry standards.

3) Implementation of career awareness and planning organized around broad industry clusters

4) Attention to the transition to postsecondary education and careers.

5) Close linkages to business and industry, with thoughtful use of local and regional labor market trends.

6) Investment in system capacity and teachers improvement.

7) Strengthening of quality control and program review processes.

Besides these the institutes may consider to adopt accreditations from different agencies that willensure an education process which yields quality engineering graduates. The frame work forimplementing CQI in engineering education can be represented in a nut shell by the followingexhibit.

\section{Conclusion}

Though in the current scenario the prospective talented students are drifting towards other non-engineering disciplines of higher education, the engineering institutes should try harder to attract them to pursue engineering courses. The only way that can be possible is by imparting quality in the graduating students. Since few years, the efforts on Quality in Teaching are being exploredby many institutes. They define and support the quality of faculty, pedagogy,learning environments, student support and other determinants contributing to successful student achievement. All the engineering institutes must put ample endeavor to implement any of the methods of achieving the desired quality in imparting engineering education. Besides CQI other methodologies include Six-Sigma, Knowledge Management, and Lean Management etc. The entire project must be well defined and proceed under the efficient leadership. The progress if monitored well then with contribution of the entire team achieving quality in engineering education will definitely turn into reality.

\section{References}

Journal Article:

Edwards, P., Huang, D., Metcalfe, L., \&Sainfort, F. (2008) Maximizing your investment in EHR. Utilizing EHRs to inform continuous quality improvement. J HealthcInf Manage, 22(1), 32-37.

Rao, P. N., Komaraiah, M., \& Reddy, P. N. (2015) A case for moocs in Indian higher education system. Journal of Engineering Education Transformations, 29(1), 15-25.

Antony, J. (2004)Some pros and cons of six sigma: An academic perspective, The TQM Magazine, 16(4), 303-306. Adina-Petruța, P., \& Roxana, S. (2014) Integrating six sigma with quality management systems for the development and continuous improvement of higher education institutions. Procedia-Social and Behavioral Sciences, 143, 643-648. 
Journal of Engineering Education Transformations, Special Issue, eISSN 2394-1707

Book:

Approval Process Handbook (2015 - 2016), AICTE. 